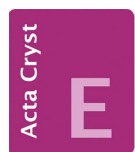

CRYSTALLOGRAPHIC COMMUNICATIONS

ISSN 2056-9890

Received 19 December 2017

Accepted 8 January 2018

Edited by C. Massera, Università di Parma, Italy

Keywords: crystal structure; hybrid coordination polymer; cadmium(II); triazole.

CCDC reference: 1810807

Supporting information: this article has supporting information at journals.iucr.org/e

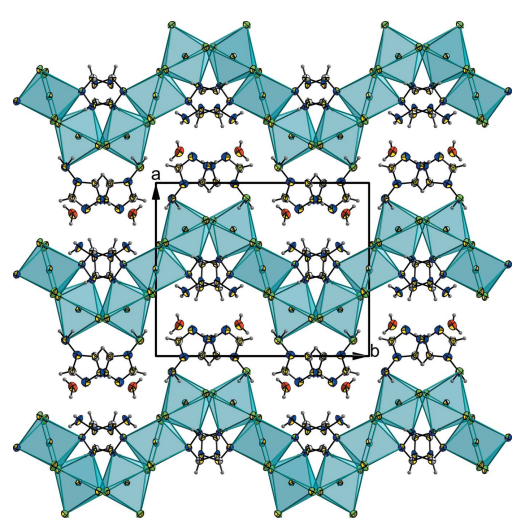

OPEN $\odot$ ACCESS

\section{A new cadmium coordination polymer based on 4-amino-4H-1,2,4-triazole}

\author{
Maha Said and Habib Boughzala*
}

Laboratoire de Matériaux et Cristallochimie, Faculté des Sciences de Tunis, Université de Tunis El Manar, 2092 Manar II Tunis, Tunisia. *Correspondence e-mail: habib.boughzala@ipein.rnu.tn

A new cadmium coordination polymer, poly[bis(4-amino-4H-1,2,4-triazolium) [bis $\left(\mu_{2}\right.$-4-amino-4H-1,2,4-triazole- $\left.\kappa^{2} N^{1}: N^{2}\right)$ tetra- $\mu_{2}$-chlorido-tetrachloridotricadmium(II)] dihydrate], $\left\{\left(\mathrm{C}_{2} \mathrm{H}_{5} \mathrm{~N}_{4}\right)_{2}\left[\mathrm{Cd}_{3} \mathrm{Cl}_{8}\left(\mathrm{C}_{2} \mathrm{H}_{4} \mathrm{~N}_{4}\right)_{2}\right] \cdot 2 \mathrm{H}_{2} \mathrm{O}\right\}_{n}$, was synthesized by the reaction of 4 -amino- $4 H-1,2,4$ triazole with cadmium(II) chloride in aqueous solution. With an unusual architecture, the crystal structure exhibits two distorted octahedral coordinations of $\mathrm{Cd}^{\mathrm{II}}$ joined by edge sharing. The first is composed by four chlorine and two $\mathrm{N}$ atoms from the triazole ligands. The second is formed by five $\mathrm{Cl}$ atoms and by one $\mathrm{N}$ atom from the triazole ligand. The charge of the resulting two-dimensional anionic framework is balanced by the organic triazole cations. The lattice water molecules form a network of hydrogen bonding. $\mathrm{N}-\mathrm{H} \cdots \mathrm{Cl}$ and $\pi-\pi$ stacking interactions are also involved in the supramolecular network stability.

\section{Chemical context}

The last decade has seen a large number of investigations of $\mathrm{Cd}^{\mathrm{II}}$ hybrid coordination polymers (HCPs). Indeed, these materials exhibit a wide variety of polymeric frameworks with attractive properties. The coordination sphere of $\mathrm{Cd}^{\mathrm{II}}$ is variable, with coordination numbers ranging from four to eight, corresponding to different geometries (tetrahedral, square planar, square pyramidal, trigonal bipyramidal, octahedral, pentagonal bipyramidal, bicapped triangular prismatic and dodecahedral; Li \& Du, 2011). Many factors should be considered in the self-assembly processes of HCPs, such as the nature of the organic ligands, temperature, $\mathrm{pH}$ values, solvents, and so on (Guo et al., 2013). The choice of the organic ligands is an important factor that greatly influences the structure and stabilization of the coordination architecture formed (Tao et al., 2000; Choi \& Jeon, 2003). In this regard, organic building units that are based on five-membered $\mathrm{N}$-heterocycles such as 1,2,4 triazole exhibit a strong and typical property of acting as bridging ligands between two metal centres. These bridges can adopt various different geometries, depending on the donor atoms of the ligand and the properties of the metal (Haasnoot et al., 2000). The reaction of 4-amino-4H-1,2,4 triazole $\left(\mathrm{NH}_{2}\right.$ trz $)$ with cadmium dichloride leads to the formation of the title two-dimensional coordination polymer.

\section{Structural commentary}

The asymmetric unit of the studied compound, completed by the atoms necessary to achieve the coordination around the $\mathrm{Cd}$ ions, is represented in Fig. 1. It comprises one and a half 
$\mathrm{Cd}^{\mathrm{II}}$ cations [with $\mathrm{Cd} 2$ occupying the special position $\left(\frac{1}{2}, \frac{1}{2}, \frac{1}{2}\right)$ ], one triazole molecule $\left(\mathrm{NH}_{2} \mathrm{trz}\right)$, one triazolium cation $\left(\mathrm{NH}_{2} \mathrm{trzH}\right)^{+}$, four chloride anions and one lattice water molecule. $\mathrm{Cd} 1$ and $\mathrm{Cd} 2$ are bridged by the coordinated triazole molecule $\left(\mathrm{NH}_{2}\right.$ trz) through atoms $\mathrm{N} 1$ and $\mathrm{N} 2$, and by the two chlorine atoms $\mathrm{Cl} 1$ and $\mathrm{Cl} 3$.

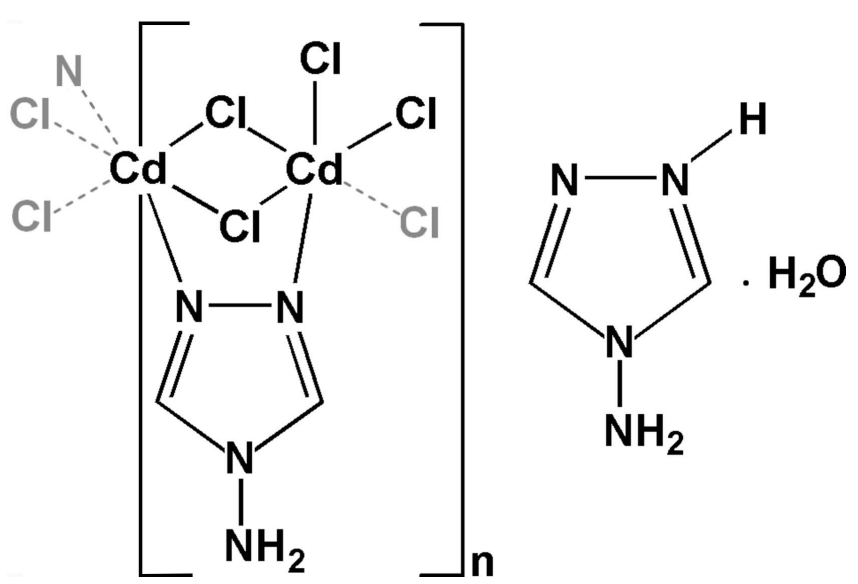

Both metals show an octahedral coordination geometry. $\mathrm{Cd} 1$ is surrounded by the five chloride anions $\mathrm{Cl} 1, \mathrm{Cl} 2, \mathrm{Cl} 3$, $\mathrm{Cl} 4, \mathrm{Cl}^{\mathrm{i}}$ [symmetry code: (i) $x, \frac{1}{2}-y, z-\frac{1}{2}$ ] and the nitrogen $\mathrm{N} 1$ of the coordinated triazole ring $\left(\mathrm{NH}_{2} \mathrm{trz}\right)$. On the other hand, $\mathrm{Cd} 2$ is bonded to four equatorial chloride anions (Cl1, $\mathrm{Cl} 3, \mathrm{Cl} 1^{\mathrm{ii}}$ and $\mathrm{Cl} 3^{\mathrm{ii}}$ ) and two axial nitrogen atoms, $\mathrm{N} 2$ and $\mathrm{N} 2{ }^{\mathrm{ii}}$, belonging to the coordinated triazole $\left(\mathrm{NH}_{2} \mathrm{trz}\right)$ and to its symmetry-related analogue, respectively [symmetry code: (ii) $1-x, 1-y, 1-z)$. As a result of the bridge formed by atoms $\mathrm{N} 1$ and $\mathrm{N} 2$ of the triazole ligand, the $\mathrm{Cd} 1 \cdots \mathrm{Cd} 2$ distance is 3.6145 (7) A. Selected geometrical parameters are summarized in Table 1, showing that the octahedron around $\mathrm{Cd} 1$ is more distorted than the one around $\mathrm{Cd} 2$.

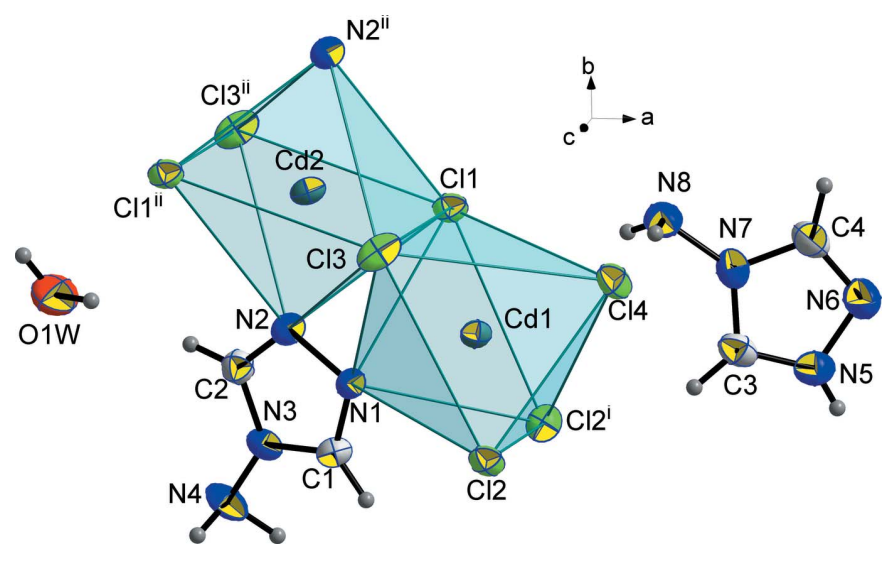

Figure 1

ORTEP of the asymmetric unit of the studied compound plus the atoms necessary to complete the coordination around the $\mathrm{Cd}$ ions. $\mathrm{Cd} 2$ is on the special position $\left(\frac{1}{2}, \frac{1}{2}, \frac{1}{2}\right)$. Displacement ellipsoids are drawn at the at the $50 \%$ probability level. [Symmetry codes: (i) $x, \frac{1}{2}-y,-\frac{1}{2}+z$; (ii) $1-x$, $1-y, 1-z$.
Table 1

Selected geometric parameters $\left(\AA{ }^{\circ}\right)$.

\begin{tabular}{lcll}
\hline $\mathrm{Cd} 1-\mathrm{N} 1$ & $2.365(4)$ & $\mathrm{Cd} 1-\mathrm{Cl} 1$ & $2.6769(14)$ \\
$\mathrm{Cd} 1-\mathrm{Cl} 4$ & $2.5120(14)$ & $\mathrm{Cd} 2-\mathrm{N} 2$ & $2.393(5)$ \\
$\mathrm{Cd} 1-\mathrm{Cl} 2$ & $2.6148(13)$ & $\mathrm{Cd} 2-\mathrm{Cl} 3$ & $2.5874(16)$ \\
$\mathrm{Cd} 1-\mathrm{Cl} 3$ & $2.6418(14)$ & $\mathrm{Cd} 2-\mathrm{Cl} 1$ & $2.6332(14)$ \\
$\mathrm{Cd} 1-\mathrm{Cl} 2^{\mathrm{i}}$ & $2.6754(13)$ & & \\
& & & \\
$\mathrm{N} 1-\mathrm{Cd} 1-\mathrm{Cl} 4$ & $174.37(11)$ & $\mathrm{Cl} 3-\mathrm{Cd} 1-\mathrm{Cl} 1$ & $84.86(4)$ \\
$\mathrm{Cl} 2-\mathrm{Cd} 1-\mathrm{Cl} 1$ & $174.60(4)$ & $\mathrm{Cl} 3-\mathrm{Cd} 2-\mathrm{Cl} 1$ & $86.85(5)$ \\
\hline
\end{tabular}

Symmetry code: (i) $x,-y+\frac{1}{2}, z-\frac{1}{2}$.

When symmetry is applied, a $\mathrm{Cd}_{3} \mathrm{Cl}_{8}\left(\mathrm{NH}_{2} \text { trz }\right)_{2}$ building block is formed. These trinuclear units are connected via the chloride ions $\mathrm{Cl} 2$ to build up infinite inorganic corrugated sheets in the $b c$ plane, stacked along the $a$-axis direction (Fig. 2). The triazolium cations $\left(\mathrm{NH}_{2} \mathrm{trzH}\right)^{+}$and the water molecules are located in the interlayer space (Fig. 3), interacting with the anionic framework by hydrogen bonds. Thus, the overall three-dimensional network consists of alternate organic-inorganic hybrid layers, responsible for the interesting behaviour of this class of materials.

\section{Supramolecular features}

The crystal structure of the title compound is mainly stabilized by hydrogen-bonding and $\pi-\pi$ stacking interactions. In particular, a number of $\mathrm{O}-\mathrm{H} \cdots \mathrm{Cl}, \mathrm{O}-\mathrm{H} \cdots \mathrm{N}, \mathrm{N}-\mathrm{H} \cdots \mathrm{O}$ and $\mathrm{N}-\mathrm{H} \cdots \mathrm{Cl}$ hydrogen bonds is present (Table 2), involving the lattice water molecules, the triazolium cations, the organic ligands and the chlorine anions. These hydrogen bonds connect the organic and inorganic moieties, leading to a self-organized, hydrated hybrid structure.

The chloride anions around $\mathrm{Cd} 1$ and $\mathrm{Cd} 2$ form hydrogen bonds both with the amine $\mathrm{H}$ atoms of the $\left(\mathrm{NH}_{2}\right.$ trz $)$ ligands and with the $\mathrm{H}$ atoms of the water molecules (Figs. 4 and 5; Table 2): $\mathrm{Cl} 1 \cdots \mathrm{H} W 2^{\mathrm{ii}}-\mathrm{O} 1 W^{\mathrm{ii}}, \quad \mathrm{Cl} 3 \cdots \mathrm{H} 4 B^{\mathrm{iii}}-\mathrm{N} 4^{\mathrm{iii}}$, $\mathrm{Cl} 4 \cdots \mathrm{H} W 1^{\mathrm{ii}}-\mathrm{O} 1 W^{\mathrm{ii}}, \mathrm{Cl} 4 \cdots \mathrm{H} 8 B-\mathrm{N} 8$, and $\mathrm{Cl} 2 \cdots \mathrm{H} 8 A^{\mathrm{iv}}-\mathrm{N} 8^{\mathrm{iv}}$ [symmetry codes: (iii) $1-x, \frac{1}{2}+y, \frac{3}{2}-z$; (iv) $x, y, 1+z$ ].

Besides forming hydrogen bonds with the chloride anions $\mathrm{Cl} 1$ and $\mathrm{Cl} 4$, the water molecules also interact with the triazole ligands and with the lattice triazolium cations, acting as

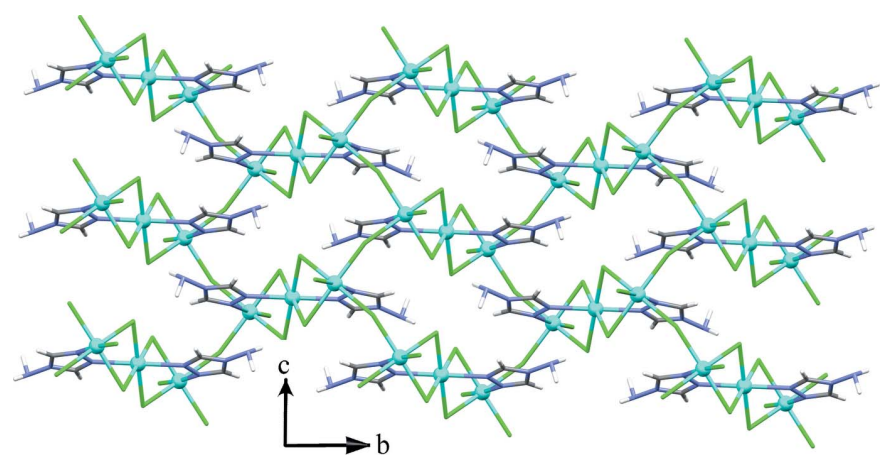

Figure 2

Crystal packing showing the two-dimensional anionic framework of the title compound. 
Table 2

Hydrogen-bond geometry $\left(\AA,^{\circ}\right)$.

\begin{tabular}{lllll}
\hline$D-\mathrm{H} \cdots A$ & $D-\mathrm{H}$ & $\mathrm{H} \cdots A$ & $D \cdots A$ & $D-\mathrm{H} \cdots A$ \\
\hline $\mathrm{O} 1 W^{\mathrm{ii}}-\mathrm{H} W 2^{\mathrm{ii}} \cdots \mathrm{Cl} 1$ & $0.86(6)$ & $2.68(7)$ & $3.239(6)$ & $124(6)$ \\
$\mathrm{N} 4^{\mathrm{iii}}-\mathrm{H} 4 B^{\mathrm{iii}} \cdots \mathrm{Cl} 3$ & $1.00(8)$ & $2.60(7)$ & $3.399(5)$ & $136(5)$ \\
$\mathrm{N} 8^{\mathrm{iv}}-\mathrm{H} 8 A^{\mathrm{iv}} \cdots \mathrm{Cl} 2$ & 0.85 & 2.64 & $3.370(5)$ & 144 \\
$\mathrm{O} 1 W^{\mathrm{ii}}-\mathrm{H} W 1^{\mathrm{ii}} \cdots \mathrm{Cl} 4$ & $0.86(7)$ & $2.67(8)$ & $3.319(6)$ & $134(8)$ \\
$\mathrm{N} 8-\mathrm{H} 8 B \cdots \mathrm{Cl} 4$ & 0.90 & 2.53 & $3.423(5)$ & 172 \\
$\mathrm{~N} 5^{\mathrm{v}}-\mathrm{H} 5^{\mathrm{v}} \cdots \mathrm{O} 1 W$ & $0.75(8)$ & $1.97(8)$ & $2.649(8)$ & $151(8)$ \\
$\mathrm{O} 1 W-\mathrm{H} W 2 \cdots \mathrm{N} 4^{\mathrm{vi}}$ & $0.86(6)$ & $2.44(6)$ & $3.247(9)$ & $157(6)$ \\
\hline
\end{tabular}

Symmetry codes: (ii) $-x+1,-y+1,-z+1$; (iii) $-x+1, y+\frac{1}{2},-z+\frac{3}{2}$; (iv) $x, y, z+1$; (v) $x-1,-y+\frac{1}{2}, z+\frac{1}{2}$; (vi) $x,-y+\frac{1}{2}, z+\frac{1}{2}$.

acceptor and donor, respectively (Fig. 6 and Table 2): $\mathrm{O} 1 W \cdots \mathrm{H} 5^{\mathrm{v}}-\mathrm{N} 5^{\mathrm{v}}$ and $\mathrm{N} 4^{\mathrm{vi}} \cdot \cdot \mathrm{H} W 2-\mathrm{O} 1 W$ [symmetry codes: (v) $x-1, \frac{1}{2}-y, \frac{1}{2}+z$; (vi) $\left.x, \frac{1}{2}-y, z+\frac{1}{2}\right]$.

Finally, the coordinated triazole rings $\left(\mathrm{NH}_{2} \mathrm{trz}\right)$ are connected along the $c$-axis direction through $\pi-\pi$ stacking interactions, with a centroid-centroid distance of 3.761 (7) $\AA$.

\section{Database survey}

Recently, a great deal of attention has been paid to the rational design and synthesis of new hybrid coordination polymers (HCPs) composed of metal ions and bridging ligands due to their fascinating structural diversity and their potential application as functional materials (Xiong et al., 2001; Liao et al., 2004; Gao et al., 2008). These coordination polymers exhibit a wide range of infinite zero- to three-dimensional frameworks with interesting structural features, which result

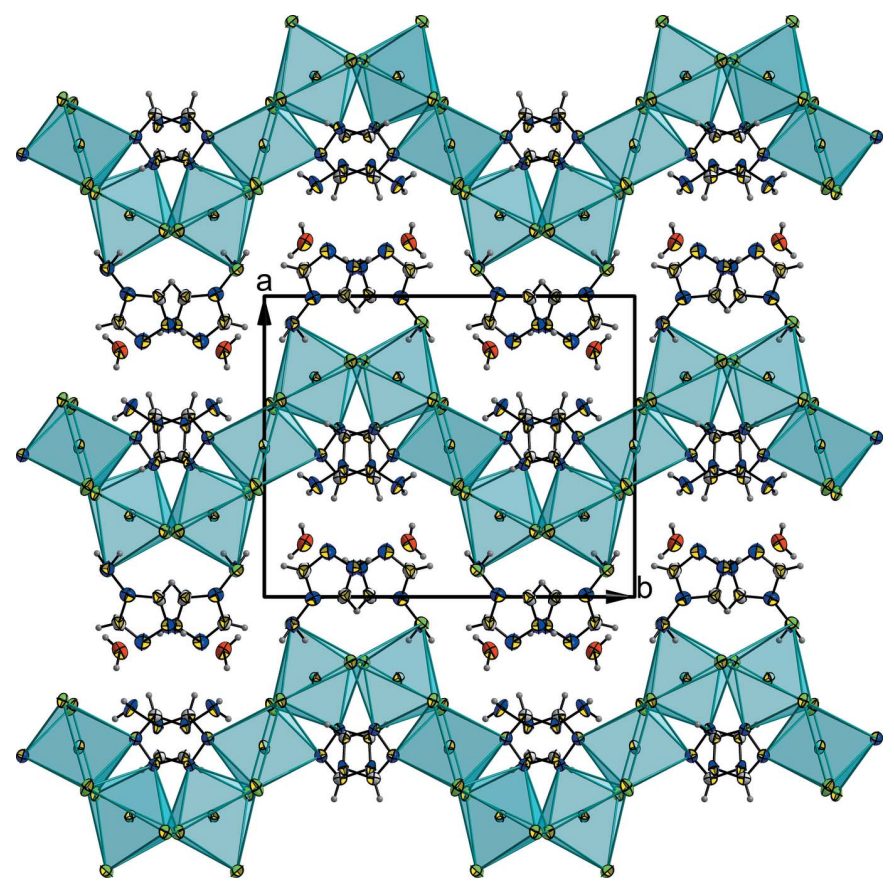

Figure 3

Corrugated anionic sheets with the non-coordinating triazolium cations and water molecules located in the interlayer space. Displacement ellipsoids are drawn at the $50 \%$ probability level.

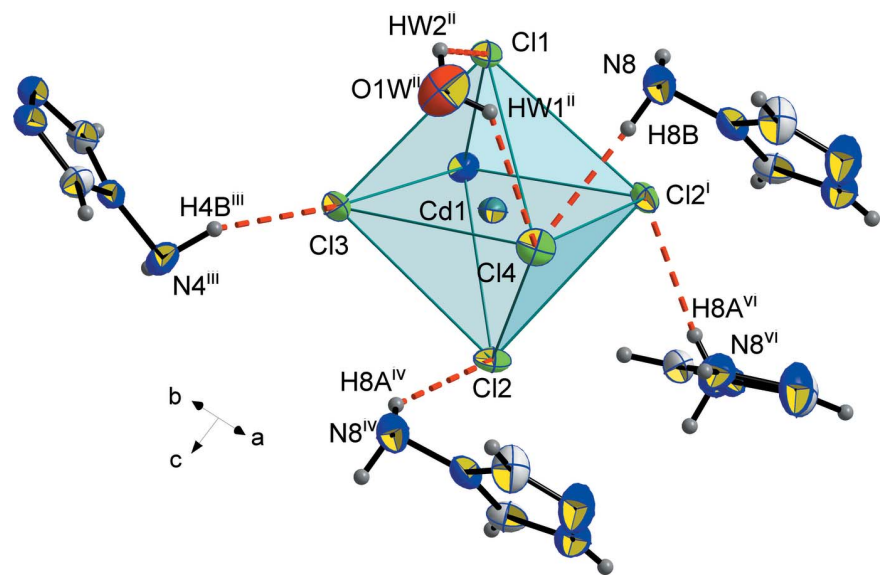

Figure 4

Hydrogen bonds (red dashed lines) involving the chloride anions around Cd1. Displacement ellipsoids are displayed at the $50 \%$ probability level. [Symmetry codes: (i) $x, \frac{1}{2}-y,-\frac{1}{2}+z$; (ii) $1-x, 1-y, 1-z$; (iii) $1-x$, $\frac{1}{2}+y, \frac{3}{2}-z$; (iv) $x, y, 1+z$; (vi) $x, \frac{1}{2}-y, \frac{1}{2}+z$.]

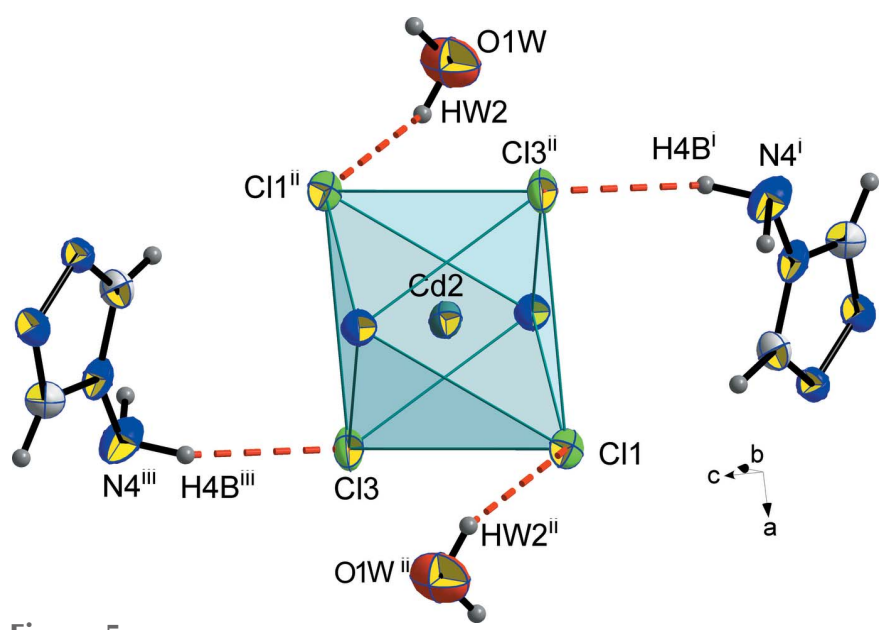

Figure 5

Hydrogen bonds (red dashed lines) involving the chloride anions around Cd2. Displacement ellipsoids are displayed at the $50 \%$ probability level. [Symmetry codes: (i) $x, \frac{1}{2}-y,-\frac{1}{2}+z$; (ii) $1-x, 1-y, 1-z$; (iii) $1-x$, $\left.\frac{1}{2}+y, \frac{3}{2}-z \cdot\right]$

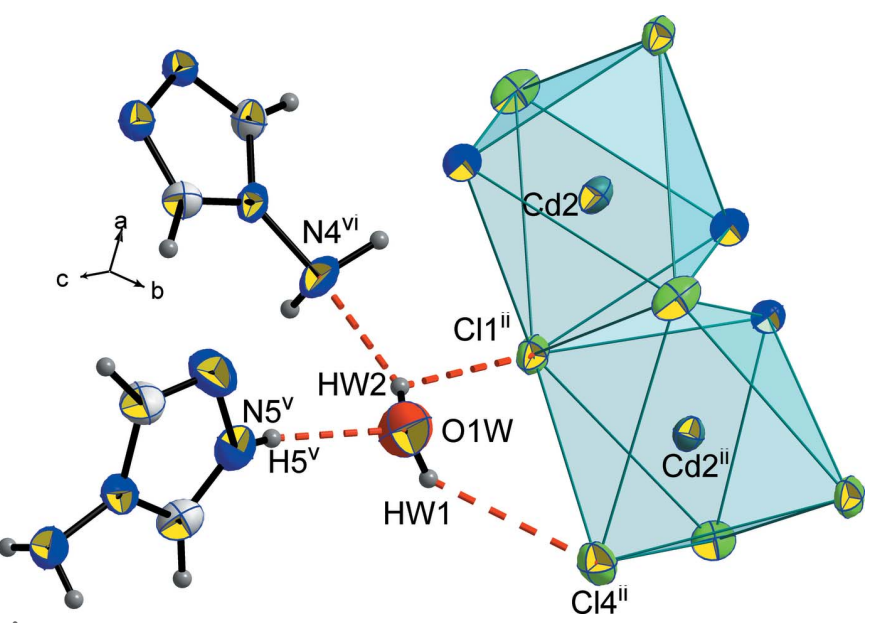

Figure 6

The hydrogen-bonding interactions around a single water molecule involving the chlorine atoms, the $\left(\mathrm{NH}_{2} \mathrm{trz}\right)$ ligand and the $\left(\mathrm{NH}_{2} \operatorname{trzH}\right)^{+}$ cation. Displacement ellipsoids are displayed at the $50 \%$ probability level. [Symmetry codes: (ii) $1-x, 1-y, 1-z ;$ (v) $-1+x, \frac{1}{2}-y, \frac{1}{2}+z$; (vi) $\left.x, \frac{1}{2}-y, \frac{1}{2}+z.\right]$ 
Table 3

Experimental details.

\begin{tabular}{|c|c|}
\hline \multicolumn{2}{|l|}{ Crystal data } \\
\hline Chemical formula & $\begin{array}{l}\left(\mathrm{C}_{2} \mathrm{H}_{5} \mathrm{~N}_{4}\right)_{2}\left[\mathrm{Cd}_{3} \mathrm{Cl}_{8}\left(\mathrm{C}_{2} \mathrm{H}_{4} \mathrm{~N}_{4}\right)_{2}\right] \cdot 2 \mathrm{H}_{2-} \\
\mathrm{O}\end{array}$ \\
\hline$M_{\mathrm{r}}$ & 995.21 \\
\hline Crystal system, space group & Monoclinic, $P 2_{1} / c$ \\
\hline Temperature $(\mathrm{K})$ & 298 \\
\hline$a, b, c(\AA)$ & 12.685 (3), 15.498 (3), 7.375 (2) \\
\hline$\beta\left({ }^{\circ}\right)$ & $97.12(3)$ \\
\hline$V\left(\AA^{6}\right)$ & $1438.6(6)$ \\
\hline$Z$ & 2 \\
\hline Radiation type & Мо $K \alpha$ \\
\hline$\mu\left(\mathrm{mm}^{-1}\right)$ & 2.98 \\
\hline Crystal size $(\mathrm{mm})$ & $0.71 \times 0.21 \times 0.21$ \\
\hline \multicolumn{2}{|l|}{ Data collection } \\
\hline Diffractometer & Enraf-Nonius CAD-4 \\
\hline Absorption correction & $\psi$ scan (North et al., 1968) \\
\hline$T_{\min }, T_{\max }$ & $0.799,1.000$ \\
\hline $\begin{array}{l}\text { No. of measured, independent and } \\
\text { observed }[I>2 \sigma(I)] \text { reflections }\end{array}$ & $3670,3136,2654$ \\
\hline$R_{\text {int }}$ & 0.032 \\
\hline$(\sin \theta / \lambda)_{\max }\left(\AA^{-1}\right)$ & 0.638 \\
\hline \multicolumn{2}{|l|}{ Refinement } \\
\hline$R\left[F^{2}>2 \sigma\left(F^{2}\right)\right], w R\left(F^{2}\right), S$ & $0.040,0.123,1.06$ \\
\hline No. of reflections & 3136 \\
\hline No. of parameters & 190 \\
\hline No. of restraints & 5 \\
\hline $\mathrm{H}$-atom treatment & $\begin{array}{l}\mathrm{H} \text { atoms treated by a mixture of } \\
\text { independent and constrained } \\
\text { refinement }\end{array}$ \\
\hline$\Delta \rho_{\max }, \Delta \rho_{\min }\left(\mathrm{e} \AA^{-3}\right)$ & $1.58,-1.99$ \\
\hline
\end{tabular}

from coordination bonding, hydrogen-bonding and aromatic $\pi-\pi$ stacking interactions as well as van der Waals forces $(\mathrm{Su}$ et al., 2003).

A search of the latest version of the Cambridge Structural Database (Version 5.38; Groom et al., 2016) based on the organic fragment '4-amino-4H-1,2,4-triazole' of the studied compound yielded 70 hits. The structure of the chlorocadmate PEPWIR (Zhai et al., 2006) is probably the nearest to that of the title compound, even if it lacks the water molecules of crystallization and the protonated triazole cations. This is probably due to the difference in the stoichiometry of the initial reagents and to the solvent used in the chemical synthesis. Two other related compounds comprising 4-amino$4 H$-1,2,4-triazole in combination with chloride ligands are the coordination polymer ROFJED (Wang et al., 2014) and the discrete complex GAVFEP (Xuan-Wen, 2005).

\section{Synthesis and crystallization}

The compound was prepared by the reaction of 4-amino-4H1,2,4 triazole and $\mathrm{CdCl}_{2} \cdot \mathrm{H}_{2} \mathrm{O}$ (molar ratio 1:1) in an equal volume of water and ethanol $(10 \mathrm{ml})$ mixed with $2 \mathrm{ml}$ of hydrochloric acid (37\%). The solution was stirred for $1 \mathrm{~h}$.
Colourless crystals suitable for X-ray diffraction were grown in two weeks by slow evaporation at room temperature.

\section{Refinement}

Crystal data, data collection and structure refinement details are summarized in Table 3. Atoms $\mathrm{H} 1, \mathrm{H} 2$ and $\mathrm{H} 3$ were placed in calculated positions and refined using a riding model: $\mathrm{C}-\mathrm{H}$ $=0.93 \AA$ with $U_{\text {iso }}(\mathrm{H})=1.2 U_{\text {eq }}(\mathrm{C})$. The other hydrogen atoms were found in the difference-Fourier map. The coordinates of $\mathrm{H} 8 A, \mathrm{H} 8 B$ and $\mathrm{H} 4 A$ of the amine terminal groups were kept fixed, with $U_{i s o}(\mathrm{H})=0.05$.

\section{Acknowledgements}

We acknowledge the assistance of the staff of the Tunisian Laboratory of Materials and Crystallography during the data collection.

\section{Funding information}

Funding for this research was provided by: Université de Tunis El Manar (Tunisia).

\section{References}

Brandenburg, K. (2006). DIAMOND. Crystal Impact GbR, Bonn, Germany.

Choi, K. Y. \& Jeon, Y. M. (2003). Inorg. Chem. Commun. 6, 1294 1296.

Enraf-Nonius (1994). CAD-4 EXPRESS. Enraf-Nonius, Delft, The Netherlands.

Gao, C., Wu, Y.-Z., Gong, H.-B., Hao, X.-P., Xu, X.-G. \& Jiang, M.-H. (2008). Inorg. Chem. Commun. 11, 985-987.

Groom, C. R., Bruno, I. J., Lightfoot, M. P. \& Ward, S. C. (2016). Acta Cryst. B72, 171-179.

Guo, F., Zhu, B., Xu, G., Zhang, M., Zhang, X. \& Zhang, J. (2013). J. Solid State Chem. 199, 42-48.

Haasnoot, J. (2000). Coord. Chem. Rev. 200-202, 131-185.

Harms, K. \& Wocadlo, S. (1995). XCAD4. Program for Processing CAD-4 Diffractometer Data. University of Marburg, Germany.

Li, C. P. \& Du, M. (2011). Inorg. Chem. Commun. 14, 502-513.

Liao, J., Lai, C., Ho, C. \& Su, C. (2004). Inorg. Chem. Commun. 7, 402-404.

North, A. C. T., Phillips, D. C. \& Mathews, F. S. (1968). Acta Cryst. A24, 351-359.

Sheldrick, G. M. (2008). Acta Cryst. A64, 112-122.

Sheldrick, G. M. (2015). Acta Cryst. C71, 3-8.

Su, Y., Goforth, A., Smith, M. \& zur Loye, H. (2003). Inorg. Chem. 42 , 5685-5692.

Tao, J., Tong, M. L. \& Chen, X. M. (2000). J. Chem. Soc. Dalton Trans. pp. 3669-3674.

Wang, P.-N., Yeh, C.-W., Tsou, C.-H., Ho, Y.-W., Lee, H.-T. \& Suen, M.-C. (2014). Inorg. Chem. Commun. 43, 70-74.

Westrip, S. P. (2010). J. Appl. Cryst. 43, 920-925.

Xiong, R.-G., You, X.-Z., Abrahams, B. F., Xue, Z. \& Che, C.-M. (2001). Angew. Chem. Int. Ed. 40, 4422-4425.

Xuan-Wen, L. (2005). Acta Cryst. E61, m1777-m1778.

Zhai, Q.-G., Wu, X.-Y., Chen, S.-M., Lu, C.-Z. \& Yang, W.-B. (2006). Cryst. Growth Des. 6, 2126-2135. 


\section{supporting information}

Acta Cryst. (2018). E74, 147-150 [https://doi.org/10.1107/S2056989018000464]

\section{A new cadmium coordination polymer based on 4-amino-4H-1,2,4-triazole}

\section{Maha Said and Habib Boughzala}

Computing details

Data collection: CAD-4 EXPRESS (Enraf-Nonius, 1994); cell refinement: CAD-4 EXPRESS (Enraf-Nonius, 1994); data reduction: XCAD4 (Harms \& Wocadlo, 1995); program(s) used to solve structure: SHELXS97 (Sheldrick, 2008); program(s) used to refine structure: SHELXL2014 (Sheldrick, 2015); molecular graphics: DIAMOND (Brandenburg, 2006); software used to prepare material for publication: publCIF (Westrip, 2010).

Poly[bis(4-amino-4H-1,2,4-triazolium) [bis $\left(\mu_{2}\right.$-4-amino-4H-1,2,4-triazole- $\left.\kappa^{2} N^{1}: N^{2}\right)$ tetra- $\mu_{2}$-chloridotetrachloridotricadmium(II)] dihydrate]

Crystal data

$\left(\mathrm{C}_{2} \mathrm{H}_{5} \mathrm{~N}_{4}\right)_{2}\left[\mathrm{Cd}_{3} \mathrm{Cl}_{8}\left(\mathrm{C}_{2} \mathrm{H}_{4} \mathrm{~N}_{4}\right)_{2}\right] \cdot 2 \mathrm{H}_{2} \mathrm{O}$

$M_{r}=995.21$

Monoclinic, $P 2_{1} / c$

$a=12.685(3) \AA$

$b=15.498(3) \AA$

$c=7.375(2) \AA$

$\beta=97.12(3)^{\circ}$

$V=1438.6(6) \AA^{3}$

$Z=2$

\section{Data collection}

Enraf-Nonius CAD-4

diffractometer

Radiation source: Enraf Nonius FR590

non-profiled $\omega / 2 \tau$ scans

Absorption correction: $\psi$ scan

(North et al., 1968)

$T_{\min }=0.799, T_{\max }=1.000$

3670 measured reflections

3136 independent reflections

Refinement

Refinement on $F^{2}$

Least-squares matrix: full

$R\left[F^{2}>2 \sigma\left(F^{2}\right)\right]=0.040$

$w R\left(F^{2}\right)=0.123$

$S=1.06$

3136 reflections

190 parameters

5 restraints

Hydrogen site location: mixed
$F(000)=956$

$D_{\mathrm{x}}=2.298 \mathrm{Mg} \mathrm{m}^{-3}$

Mo $K \alpha$ radiation, $\lambda=0.71073 \AA$

Cell parameters from 25 reflections

$\theta=10-15^{\circ}$

$\mu=2.98 \mathrm{~mm}^{-1}$

$T=298 \mathrm{~K}$

Prism, colourless

$0.71 \times 0.21 \times 0.21 \mathrm{~mm}$

2654 reflections with $I>2 \sigma(I)$

$R_{\text {int }}=0.032$

$\theta_{\max }=27.0^{\circ}, \theta_{\min }=2.1^{\circ}$

$h=-16 \rightarrow 16$

$k=-19 \rightarrow 1$

$l=-9 \rightarrow 1$

2 standard reflections every $120 \mathrm{~min}$ intensity decay: $8 \%$

$\mathrm{H}$ atoms treated by a mixture of independent and constrained refinement

$w=1 /\left[\sigma^{2}\left(F_{\mathrm{o}}^{2}\right)+(0.0848 P)^{2}+1.0345 P\right]$

where $P=\left(F_{\mathrm{o}}{ }^{2}+2 F_{\mathrm{c}}{ }^{2}\right) / 3$

$(\Delta / \sigma)_{\max }=0.001$

$\Delta \rho_{\max }=1.58 \mathrm{e} \AA^{-3}$

$\Delta \rho_{\min }=-1.99$ e $\AA^{-3}$ 
Extinction correction: SHELXL2014

(Sheldrick, 2015),

$\mathrm{Fc}^{*}=\mathrm{kFc}\left[1+0.001 \times \mathrm{Fc}^{2} \lambda^{3} / \sin (2 \theta)\right]^{-1 / 4}$

Extinction coefficient: 0.0041 (7)

\section{Special details}

Geometry. All esds (except the esd in the dihedral angle between two 1.s. planes) are estimated using the full covariance matrix. The cell esds are taken into account individually in the estimation of esds in distances, angles and torsion angles; correlations between esds in cell parameters are only used when they are defined by crystal symmetry. An approximate (isotropic) treatment of cell esds is used for estimating esds involving l.s. planes.

Refinement. Refinement of $\mathrm{F}^{2}$ against ALL reflections. The weighted R-factor $\mathrm{wR}$ and goodness of fit $\mathrm{S}$ are based on $\mathrm{F}^{2}$, conventional R-factors $R$ are based on $F$, with $F$ set to zero for negative $F^{2}$. The threshold expression of $F^{2}>2 \operatorname{sigma}\left(F^{2}\right)$ is used only for calculating R-factors(gt) etc. and is not relevant to the choice of reflections for refinement. R-factors based on $\mathrm{F}^{2}$ are statistically about twice as large as those based on F, and R- factors based on ALL data will be even larger.

Fractional atomic coordinates and isotropic or equivalent isotropic displacement parameters $\left(\AA^{2}\right)$

\begin{tabular}{lllll}
\hline & $x$ & $y$ & $z$ & $U_{\text {iso }}^{*} / U_{\text {eq }}$ \\
\hline Cd1 & $0.72922(3)$ & $0.36225(2)$ & $0.60671(4)$ & $0.02384(16)$ \\
Cd2 & 0.5000 & 0.5000 & 0.5000 & $0.02726(18)$ \\
C11 & $0.65520(10)$ & $0.45547(8)$ & $0.31268(16)$ & $0.0291(3)$ \\
C12 & $0.78296(10)$ & $0.26723(9)$ & $0.89644(16)$ & $0.0307(3)$ \\
C13 & $0.63396(12)$ & $0.47886(9)$ & $0.79095(17)$ & $0.0379(3)$ \\
C14 & $0.91217(11)$ & $0.42626(9)$ & $0.6250(2)$ & $0.0369(3)$ \\
N1 & $0.5591(3)$ & $0.2978(3)$ & $0.5599(6)$ & $0.0271(9)$ \\
N2 & $0.4709(4)$ & $0.3474(3)$ & $0.5015(6)$ & $0.0300(9)$ \\
N3 & $0.4303(4)$ & $0.2130(3)$ & $0.4573(6)$ & $0.0284(9)$ \\
N4 & $0.3704(5)$ & $0.1390(3)$ & $0.3952(8)$ & $0.0427(12)$ \\
N5 & $1.0964(5)$ & $0.2603(4)$ & $0.1439(8)$ & $0.0406(11)$ \\
N6 & $1.1503(5)$ & $0.3284(4)$ & $0.0808(9)$ & $0.0552(15)$ \\
N7 & $0.9940(4)$ & $0.3688(3)$ & $0.1463(6)$ & $0.0341(10)$ \\
N8 & $0.9074(4)$ & $0.4233(3)$ & $0.1599(6)$ & $0.0388(11)$ \\
C1 & $0.5314(4)$ & $0.2172(3)$ & $0.5326(7)$ & $0.0301(10)$ \\
H1 & 0.5755 & 0.1699 & 0.5613 & $0.036^{*}$ \\
C2 & $0.3953(4)$ & $0.2949(4)$ & $0.4392(8)$ & $0.0326(11)$ \\
H2 & 0.3273 & 0.3114 & 0.3895 & $0.039^{*}$ \\
C3 & $1.0035(5)$ & $0.2844(4)$ & $0.1816(8)$ & $0.0371(12)$ \\
H3 & 0.9528 & 0.2493 & 0.2253 & $0.044^{*}$ \\
C4 & $1.0865(6)$ & $0.3931(4)$ & $0.0848(11)$ & $0.0481(16)$ \\
O1W & $0.1792(5)$ & $0.3900(4)$ & $0.5653(10)$ & $0.0663(15)$ \\
H4 & $1.103(5)$ & $0.450(4)$ & $0.066(9)$ & $0.038(17)^{*}$ \\
H5 & $1.127(6)$ & $0.219(5)$ & $0.158(11)$ & $0.05(2)^{*}$ \\
H8A & 0.8524 & 0.4002 & 0.1015 & $0.050^{*}$ \\
H8B & 0.9153 & 0.4271 & 0.2827 & $0.050^{*}$ \\
H4A & 0.4298 & 0.0977 & 0.3612 & $0.050^{*}$ \\
H4B & $0.350(6)$ & $0.120(4)$ & $0.516(11)$ & $0.045(19)^{*}$ \\
HW2 & $0.237(4)$ & $0.396(5)$ & $0.638(9)$ & $0.07(3)^{*}$ \\
HW1 & $0.142(6)$ & $0.435(4)$ & $0.580(14)$ & $0.11(4)^{*}$ \\
& & & &
\end{tabular}


Atomic displacement parameters $\left(\AA^{2}\right)$

\begin{tabular}{lllllll}
\hline & $U^{11}$ & $U^{22}$ & $U^{33}$ & $U^{12}$ & $U^{13}$ & $U^{23}$ \\
\hline Cd1 & $0.0255(2)$ & $0.0249(2)$ & $0.0204(2)$ & $0.00043(12)$ & $0.00023(14)$ & $0.00166(12)$ \\
Cd2 & $0.0335(3)$ & $0.0216(3)$ & $0.0261(3)$ & $0.00527(19)$ & $0.0016(2)$ & $0.00027(18)$ \\
C11 & $0.0363(6)$ & $0.0285(6)$ & $0.0229(5)$ & $0.0036(5)$ & $0.0046(5)$ & $0.0044(4)$ \\
C12 & $0.0347(6)$ & $0.0341(7)$ & $0.0227(6)$ & $-0.0022(5)$ & $0.0013(5)$ & $0.0100(5)$ \\
C13 & $0.0507(8)$ & $0.0372(7)$ & $0.0235(6)$ & $0.0143(6)$ & $-0.0041(5)$ & $-0.0085(5)$ \\
C14 & $0.0327(7)$ & $0.0366(7)$ & $0.0410(7)$ & $-0.0101(5)$ & $0.0027(5)$ & $-0.0007(6)$ \\
N1 & $0.026(2)$ & $0.026(2)$ & $0.029(2)$ & $0.0019(16)$ & $0.0020(16)$ & $0.0019(16)$ \\
N2 & $0.032(2)$ & $0.029(2)$ & $0.028(2)$ & $0.0029(18)$ & $0.0022(17)$ & $-0.0003(17)$ \\
N3 & $0.040(2)$ & $0.025(2)$ & $0.022(2)$ & $-0.0059(18)$ & $0.0095(17)$ & $-0.0032(15)$ \\
N4 & $0.054(3)$ & $0.034(3)$ & $0.042(3)$ & $-0.020(2)$ & $0.011(2)$ & $-0.009(2)$ \\
N5 & $0.046(3)$ & $0.034(3)$ & $0.041(3)$ & $-0.001(2)$ & $0.003(2)$ & $0.000(2)$ \\
N6 & $0.054(3)$ & $0.047(3)$ & $0.071(4)$ & $0.002(3)$ & $0.031(3)$ & $0.005(3)$ \\
N7 & $0.035(2)$ & $0.043(3)$ & $0.025(2)$ & $-0.0025(19)$ & $0.0072(18)$ & $-0.0042(18)$ \\
N8 & $0.044(3)$ & $0.034(2)$ & $0.040(3)$ & $-0.002(2)$ & $0.009(2)$ & $-0.006(2)$ \\
C1 & $0.034(3)$ & $0.025(2)$ & $0.031(3)$ & $0.003(2)$ & $0.002(2)$ & $0.002(2)$ \\
C2 & $0.030(3)$ & $0.034(3)$ & $0.034(3)$ & $-0.001(2)$ & $0.003(2)$ & $-0.001(2)$ \\
C3 & $0.042(3)$ & $0.037(3)$ & $0.031(3)$ & $-0.007(2)$ & $0.001(2)$ & $0.007(2)$ \\
C4 & $0.051(4)$ & $0.037(3)$ & $0.061(4)$ & $-0.007(3)$ & $0.023(3)$ & $0.000(3)$ \\
O1W & $0.057(3)$ & $0.043(3)$ & $0.095(5)$ & $-0.011(3)$ & $-0.002(3)$ & $-0.002(3)$ \\
& & & & & & \\
\hline
\end{tabular}

Geometric parameters $\left(A,{ }^{\circ}\right)$

\begin{tabular}{llll}
\hline $\mathrm{Cd} 1-\mathrm{N} 1$ & $2.365(4)$ & $\mathrm{N} 4-\mathrm{H} 4 \mathrm{~A}$ & 1.0400 \\
$\mathrm{Cd} 1-\mathrm{Cl} 4$ & $2.5120(14)$ & $\mathrm{N} 4-\mathrm{H} 4 \mathrm{~B}$ & $1.01(8)$ \\
$\mathrm{Cd} 1-\mathrm{Cl} 2$ & $2.6148(13)$ & $\mathrm{N} 5-\mathrm{C} 3$ & $1.299(9)$ \\
$\mathrm{Cd} 1-\mathrm{Cl} 3$ & $2.6418(14)$ & $\mathrm{N} 5-\mathrm{N} 6$ & $1.369(8)$ \\
$\mathrm{Cd} 1-\mathrm{Cl} 2^{\mathrm{i}}$ & $2.6754(13)$ & $\mathrm{N} 5-\mathrm{H} 5$ & $0.75(8)$ \\
$\mathrm{Cd} 1-\mathrm{Cl1}$ & $2.6769(14)$ & $\mathrm{N} 6-\mathrm{C} 4$ & $1.292(9)$ \\
$\mathrm{Cd} 2-\mathrm{N} 2^{\mathrm{ii}}$ & $2.393(5)$ & $\mathrm{N} 7-\mathrm{C} 3$ & $1.336(7)$ \\
$\mathrm{Cd} 2-\mathrm{N} 2$ & $2.393(5)$ & $\mathrm{N} 7-\mathrm{C} 4$ & $1.362(8)$ \\
$\mathrm{Cd} 2-\mathrm{Cl} 3$ & $2.5874(16)$ & $\mathrm{N} 7-\mathrm{N} 8$ & $1.399(7)$ \\
$\mathrm{Cd} 2-\mathrm{Cl} 3^{\mathrm{ii}}$ & $2.5875(16)$ & $\mathrm{N} 8-\mathrm{H} 8 \mathrm{~A}$ & 0.850 \\
$\mathrm{Cd} 2-\mathrm{Cl} 1$ & $2.6332(14)$ & $\mathrm{N} 8-\mathrm{H} 8 \mathrm{~B}$ & 0.900 \\
$\mathrm{Cd} 2-\mathrm{Cl1}{ }^{\mathrm{ii}}$ & $2.6333(14)$ & $\mathrm{C} 1-\mathrm{H} 1$ & 0.9300 \\
$\mathrm{~N} 1-\mathrm{C} 1$ & $1.306(7)$ & $\mathrm{C} 2-\mathrm{H} 2$ & 0.9300 \\
$\mathrm{~N} 1-\mathrm{N} 2$ & $1.382(6)$ & $\mathrm{C} 3-\mathrm{H} 3$ & 0.9300 \\
$\mathrm{~N} 2-\mathrm{C} 2$ & $1.297(7)$ & $\mathrm{C} 4-\mathrm{H} 4$ & $0.92(6)$ \\
$\mathrm{N} 3-\mathrm{C} 1$ & $1.335(7)$ & $\mathrm{O} 1 \mathrm{~W}-\mathrm{HW} 2$ & $0.862(10)$ \\
$\mathrm{N} 3-\mathrm{C} 2$ & $1.346(7)$ & $\mathrm{O} 1 \mathrm{~W}-\mathrm{HW} 1$ & $0.857(10)$ \\
$\mathrm{N} 3-\mathrm{N} 4$ & $1.419(6)$ & & \\
& & & $115.6(3)$ \\
$\mathrm{N} 1-\mathrm{Cd} 1-\mathrm{Cl} 4$ & $174.37(11)$ & $\mathrm{N} 1-\mathrm{N} 2-\mathrm{Cd} 2$ & $106.4(4)$ \\
$\mathrm{C} 14-\mathrm{Cd} 1-\mathrm{Cl} 3$ & $100.40(5)$ & $\mathrm{C} 1-\mathrm{N} 3-\mathrm{C} 2$ & $128.4(5)$ \\
$\mathrm{C} 12-\mathrm{Cd} 1-\mathrm{Cl3}$ & $93.13(5)$ & $\mathrm{C} 1-\mathrm{N} 3-\mathrm{N} 4$ & $125.0(5)$ \\
$\mathrm{N} 1-\mathrm{Cd} 1-\mathrm{Cl2}{ }^{\mathrm{i}}$ & $83.80(11)$ & $\mathrm{C} 2-\mathrm{N} 3-\mathrm{N} 4$ &
\end{tabular}




\begin{tabular}{|c|c|c|c|}
\hline $\mathrm{Cl} 4-\mathrm{Cd} 1-\mathrm{Cl} 2^{\mathrm{i}}$ & $91.57(5)$ & $\mathrm{N} 3-\mathrm{N} 4-\mathrm{H} 4 \mathrm{~A}$ & 102.00 \\
\hline $\mathrm{Cl} 2-\mathrm{Cd} 1-\mathrm{Cl} 2^{\mathrm{i}}$ & $89.53(3)$ & N3-N4-H4B & $98(4)$ \\
\hline $\mathrm{N} 1-\mathrm{Cd} 1-\mathrm{Cl} 1$ & $83.54(11)$ & $\mathrm{H} 4 \mathrm{~A}-\mathrm{N} 4-\mathrm{H} 4 \mathrm{~B}$ & 108.00 \\
\hline $\mathrm{Cl} 4-\mathrm{Cd} 1-\mathrm{Cl} 1$ & $93.40(5)$ & $\mathrm{C} 3-\mathrm{N} 5-\mathrm{N} 6$ & $110.8(5)$ \\
\hline $\mathrm{Cl} 2-\mathrm{Cd} 1-\mathrm{Cl} 1$ & $174.60(4)$ & $\mathrm{C} 3-\mathrm{N} 5-\mathrm{H} 5$ & $133(6)$ \\
\hline $\mathrm{Cl} 3-\mathrm{Cd} 1-\mathrm{Cl} 1$ & $84.86(4)$ & $\mathrm{N} 6-\mathrm{N} 5-\mathrm{H} 5$ & $116(6)$ \\
\hline $\mathrm{Cl} 2 \mathrm{i}-\mathrm{Cd} 1-\mathrm{Cl} 1$ & $91.39(4)$ & $\mathrm{C} 4-\mathrm{N} 6-\mathrm{N} 5$ & $104.5(6)$ \\
\hline $\mathrm{N} 2{ }^{\mathrm{ii}}-\mathrm{Cd} 2-\mathrm{Cl} 3$ & $92.46(11)$ & $\mathrm{C} 3-\mathrm{N} 7-\mathrm{C} 4$ & $106.0(5)$ \\
\hline $\mathrm{N} 2-\mathrm{Cd} 2-\mathrm{Cl} 3$ & $87.54(12)$ & $\mathrm{C} 3-\mathrm{N} 7-\mathrm{N} 8$ & $129.0(5)$ \\
\hline $\mathrm{N} 2^{\mathrm{ii}}-\mathrm{Cd} 2-\mathrm{Cl}^{\mathrm{ii}}$ & $87.54(11)$ & $\mathrm{C} 4-\mathrm{N} 7-\mathrm{N} 8$ & $125.0(5)$ \\
\hline $\mathrm{N} 2-\mathrm{Cd} 2-\mathrm{Cl}^{3 i}$ & $92.46(12)$ & $\mathrm{N} 7-\mathrm{N} 8-\mathrm{H} 8 \mathrm{~A}$ & 108.00 \\
\hline $\mathrm{N} 2{ }^{\mathrm{ii}}-\mathrm{Cd} 2-\mathrm{Cl} 1$ & $97.50(11)$ & $\mathrm{N} 7-\mathrm{N} 8-\mathrm{H} 8 \mathrm{~B}$ & 97.00 \\
\hline $\mathrm{N} 2-\mathrm{Cd} 2-\mathrm{Cl} 1$ & $82.50(11)$ & $\mathrm{H} 8 \mathrm{~A}-\mathrm{N} 8-\mathrm{H} 8 \mathrm{~B}$ & 121.00 \\
\hline $\mathrm{Cl} 3-\mathrm{Cd} 2-\mathrm{Cl} 1$ & $86.85(5)$ & $\mathrm{N} 1-\mathrm{C} 1-\mathrm{N} 3$ & $109.7(5)$ \\
\hline $\mathrm{Cl}_{3}{ }^{\mathrm{ii}}-\mathrm{Cd} 2-\mathrm{Cl} 1$ & $93.15(5)$ & $\mathrm{N} 1-\mathrm{C} 1-\mathrm{H} 1$ & 125.2 \\
\hline $\mathrm{N} 2^{\mathrm{ii}}-\mathrm{Cd} 2-\mathrm{Cl} 1^{\mathrm{ii}}$ & $82.50(11)$ & $\mathrm{N} 3-\mathrm{C} 1-\mathrm{H} 1$ & 125.2 \\
\hline $\mathrm{N} 2-\mathrm{Cd} 2-\mathrm{Cl}^{1 \mathrm{ii}}$ & $97.50(11)$ & $\mathrm{N} 2-\mathrm{C} 2-\mathrm{N} 3$ & $109.7(5)$ \\
\hline $\mathrm{Cl} 3-\mathrm{Cd} 2-\mathrm{Cl}^{\mathrm{ii}}$ & $93.15(5)$ & $\mathrm{N} 2-\mathrm{C} 2-\mathrm{H} 2$ & 125.1 \\
\hline $\mathrm{Cl} 3^{\mathrm{ii}}-\mathrm{Cd} 2-\mathrm{Cl}{ }^{\mathrm{ii}}$ & $86.85(5)$ & $\mathrm{N} 3-\mathrm{C} 2-\mathrm{H} 2$ & 125.1 \\
\hline $\mathrm{Cd} 2-\mathrm{Cl} 1-\mathrm{Cd} 1$ & $85.79(4)$ & $\mathrm{N} 5-\mathrm{C} 3-\mathrm{N} 7$ & $107.6(5)$ \\
\hline $\mathrm{Cd} 1-\mathrm{Cl} 2-\mathrm{Cd} 1^{\mathrm{iii}}$ & $146.79(5)$ & $\mathrm{N} 5-\mathrm{C} 3-\mathrm{H} 3$ & 126.2 \\
\hline $\mathrm{Cd} 2-\mathrm{Cl} 3-\mathrm{Cd} 1$ & $87.44(4)$ & $\mathrm{N} 7-\mathrm{C} 3-\mathrm{H} 3$ & 126.2 \\
\hline $\mathrm{C} 1-\mathrm{N} 1-\mathrm{N} 2$ & $107.0(4)$ & $\mathrm{N} 6-\mathrm{C} 4-\mathrm{N} 7$ & $111.1(6)$ \\
\hline $\mathrm{C} 1-\mathrm{N} 1-\mathrm{Cd} 1$ & $130.5(3)$ & $\mathrm{N} 6-\mathrm{C} 4-\mathrm{H} 4$ & $126(4)$ \\
\hline $\mathrm{N} 2-\mathrm{N} 1-\mathrm{Cd} 1$ & $120.0(3)$ & $\mathrm{N} 7-\mathrm{C} 4-\mathrm{H} 4$ & $122(4)$ \\
\hline $\mathrm{C} 2-\mathrm{N} 2-\mathrm{N} 1$ & $107.2(4)$ & HW2- $\mathrm{O} 1 \mathrm{~W}-\mathrm{HW} 1$ & $106(3)$ \\
\hline $\mathrm{C} 2-\mathrm{N} 2-\mathrm{Cd} 2$ & $136.6(4)$ & & \\
\hline
\end{tabular}

Symmetry codes: (i) $x,-y+1 / 2, z-1 / 2$; (ii) $-x+1,-y+1,-z+1$; (iii) $x,-y+1 / 2, z+1 / 2$.

Hydrogen-bond geometry $\left(\AA,{ }^{\circ}\right)$

\begin{tabular}{|c|c|c|c|c|}
\hline$D-\mathrm{H} \cdots A$ & $D-\mathrm{H}$ & $\mathrm{H} \cdots A$ & $D \cdots A$ & $D-\mathrm{H} \cdots A$ \\
\hline $\mathrm{O} 1 W^{\mathrm{ii}}-\mathrm{H} W 2^{\mathrm{ii} \ldots \mathrm{Cl} 1}$ & $0.86(6)$ & $2.68(7)$ & $3.239(6)$ & $124(6)$ \\
\hline $\mathrm{N} 4^{\mathrm{iv}}-\mathrm{H} 4 B^{\mathrm{iv}} \cdots \mathrm{Cl} 3$ & $1.00(8)$ & $2.60(7)$ & $3.399(5)$ & $136(5)$ \\
\hline $\mathrm{N} 8^{v}-\mathrm{H} 8 A^{\mathrm{v} \cdots} \mathrm{Cl} 2$ & 0.85 & 2.64 & $3.370(5)$ & 144 \\
\hline $\mathrm{O} 1 W^{\mathrm{ii}}-\mathrm{H} W 1^{\mathrm{ii} \ldots} \mathrm{Cl} 4$ & $0.86(7)$ & $2.67(8)$ & $3.319(6)$ & $134(8)$ \\
\hline $\mathrm{N} 8-\mathrm{H} 8 B \cdots \mathrm{Cl} 4$ & 0.90 & 2.53 & $3.423(5)$ & 172 \\
\hline $\mathrm{N} 5^{\mathrm{vi}}-\mathrm{H} 5^{\mathrm{vi} \ldots \mathrm{O}} 1 W$ & $0.75(8)$ & $1.97(8)$ & $2.649(8)$ & $151(8)$ \\
\hline $\mathrm{O} 1 W-\mathrm{H} W 2 \cdots \mathrm{N} 4^{\mathrm{iii}}$ & $0.86(6)$ & $2.44(6)$ & $3.247(9)$ & $157(6)$ \\
\hline
\end{tabular}

Symmetry codes: (ii) $-x+1,-y+1,-z+1$; (iii) $x,-y+1 / 2, z+1 / 2$; (iv) $-x+1, y+1 / 2,-z+3 / 2$; (v) $x, y, z+1$; (vi) $x-1,-y+1 / 2, z+1 / 2$. 